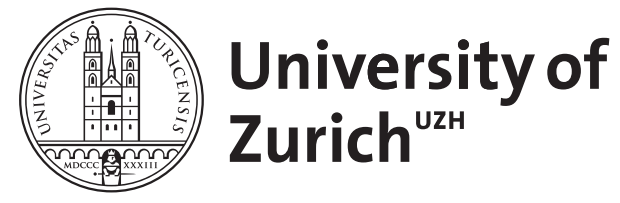

Zurich Open Repository and Archive

University of Zurich

University Library

Strickhofstrasse 39

CH-8057 Zurich

www.zora.uzh.ch

Year: 2016

\title{
Gewalt, Sinn und Verletzlichkeit
}

Meyer, Katrin

Posted at the Zurich Open Repository and Archive, University of Zurich

ZORA URL: https://doi.org/10.5167/uzh-170973

Journal Article

Published Version

Originally published at:

Meyer, Katrin (2016). Gewalt, Sinn und Verletzlichkeit. Deutsche Zeitschrift für Philosophie, 64(1):150154. 


\section{Katrin Meyer* \\ Gewalt, Sinn und Verletzlichkeit}

DOI 10.1515/dzph-2016-0011

Michael Staudigl. Phänomenologie der Gewalt, Cham, Heidelberg u. New York, Springer, 2015, 302 S.

Es gibt eine lange Tradition in der politischen Theorie und normativen Sozialphilosophie, die sich von Hannah Arendt bis zu Thomas Hobbes zurückverfolgen lässt, in der Gewalt als Ausdruck eines anomischen, sinnlosen und die politische Ordnung bedrohenden Phänomens in den Blick kommt. Gegen diese Konzeptionalisierung von Gewalt als destruktiver Kraft wendet sich Michael Staudigl in seiner Wiener Habilitationsschrift Phänomenologie der Gewalt, indem er die These vertritt, dass es nicht angemessen sei, Gewalt nur als Mangel oder Defizienz zu denken. Gewalt sei vielmehr ein „irreduzibles soziales Phänomen“ (7) und müsse in ihrer eigenen sozialen Sinnhaftigkeit erschlossen werden. Staudigl beruft sich dabei explizit auf jene aktuellen Forschungsansätze, die sich mit Phänomenen „negativer Sozialität“ (6) beschäftigen und diese in ihrem eigenen Recht erfassen wollen. Er versteht Gewalt entsprechend als ein Phänomen, das sich in einer Vielzahl von Ausdrucksformen realisiert und dennoch durch einen gemeinsamen „Erfahrungssinn“ (150) in seiner Einheit fassbar werden kann. Von der Freilegung dieses Sinns erhofft er sich auch Rückschlüsse darauf, wie Sozialität und Subjektivität zu fassen sind, wenn wir Gewalt als sozial unaufhebbar denken.

Im Zentrum seiner Untersuchung steht die These, dass Gewalt durch eine Sinnerfahrung bestimmt wird, die den Gewalt Ausübenden, den Gewalt Erleidenden und den Gewalt bezeugenden Dritten gemeinsam ist, und die in ihrer allgemeinsten Form mit „Verletzlichkeit“ zu tun hat, die in der Gewalt „auf dem Spiel“ steht (43). Verletzung in ihren verschiedenen phänomenalen Ausprägungen der „Verletzlichkeit, Verletzungsmacht und Sensibilität für die Verletzlichkeit anderer“ (189) konstituiert entsprechend den phänomenalen „Kern“ (ebd.) der Gewalt. Nach Staudigl können wir Gewalt nur verstehen, wenn wir erkennen, dass ein Subjekt der Gewalt ausgesetzt werden kann, weil es verletzungsoffen ist. Im Licht dieser Erkenntnis verändert sich auch das Konzept der Subjektivität. Dieses kann nicht mehr, wie in der klassischen Phänomenologie angelegt, als souveräner Grund subjektiver Phänomengenese gedacht werden, sondern es

*Kontakt: Katrin Meyer, Katrin.Meyer@unibas.ch 
muss in seiner Prekarität und seinem Scheitern anerkannt werden, in denen erst seine Verletzlichkeit gründet.

Staudigls Verständnis von Gewalt steht ganz eindeutig in der Tradition von Emmanuel Lévinas. Er definiert Gewalt zu Beginn seiner Untersuchung als „Verletzung der Andersheit des Anderen“ (43) und situiert Gewalt damit grundsätzlich in einem ethisch-relationalen Sinnzusammenhang, ohne dass dieser Ansatz allerdings in Auseinandersetzung mit konkurrierenden Gewaltdefinitionen weiter begründet würde. An Lévinas’ Bestimmung der Gewalt als Vernichtung der „Andersheit des Anderen“ (122) betont Staudigl, dass sie uns die Frage abnötige, wie Gewalt angesichts des ethischen Anspruchs, unter den mich der Andere zwinge, überhaupt möglich sei. Diese ethische Verpflichtung durch den Anderen, die von Lévinas als „gute Gewalt“ (125) definiert wird, bleibt gleichwohl eine Gewalt, insofern sie mich „anklagt, verfolgt und traumatisiert“ und zur Geisel nimmt (125). Staudigl folgert daraus, dass intersubjektive Beziehungen von einer Ambivalenz durchzogen seien, die erklärt, warum die Verpflichtung des Anderen unerfüllt bleiben und Gewalt faktisch möglich werden könne. Gewalt vollzieht sich demnach immer dann, wenn wir dem Appell des Anderen gegenüber indifferent sind oder seinen Anspruch auf Anerkennung seiner Andersheit negieren. Staudigl betont an verschiedenen Stellen seines Buches, dass Gewalt in allen ihren Spielarten auf dieser Möglichkeit der Indifferenz und Negation beruhe und ohne sie undenkbar sei (128, 237, 239). Die Rekonstruktion der „intersubjektiven Konstitutionsbedingungen der Sinnförmigkeit solcher Gewalt“ (230) könne diese Zusammenhänge freilegen.

Die Frage nach den „Möglichkeitsbedingungen“ (212) der Gewalt ist eine in den verschiedenen Kapiteln des Buches immer wiederkehrende. Allerdings birgt sie eine Zweideutigkeit, die mit dem Begriff der Möglichkeit verbunden ist. In welchen Logiken ist sie gefasst, welche Phänomene will sie erhellen? Ganz offensichtlich ist Staudigl nicht auf eine kausale Erklärungsfigur aus, die nach den sozialen Ursachen oder Bedingungen von Gewalt fragt, da er diese Perspektive gleich zu Beginn seines Buches für eine phänomenologische Theorie der Gewalt als inadäquat zurückweist (7). Die Ermöglichungsbedingungen der Gewalt verweisen vielmehr auf ein Selbstverhältnis der Subjekte, das eng an leibhafte Erfahrungen gebunden ist und mit der oben genannten Erfahrung der Verletzlichkeit zusammenhängt. Es handelt sich also um eine phänomenologische Möglichkeit in Bezug auf die Sinnhaftigkeit von Gewalt, oder, anders formuliert: Nur weil das Subjekt für Gewalt offen ist, ist interpersonale Gewalt überhaupt möglich. Die Möglichkeit der Gewalt gründet demnach in den subjektiven „Chiasmen leibkörperlichen Existierens“ (156), in denen sich Erfahrungen der Nicht-Identität von Körper und Leib niederschlagen. Damit einher geht die Erfahrung der Nicht-Kongruenz des Subjekts mit sich selbst, das versteht, dass es als leibhaftes nie mit 
sich identisch und von anderen unabhängig sein kann, sondern dass es in Beziehungen verstrickt, durch andere verletzbar und selbst zur Verletzung anderer fähig ist. Diese in der Leibhaftigkeit der Existenz begründete Möglichkeit der Gewalt gilt demnach grundsätzlich für jedes Subjekt und ist nicht danach unterschieden, welche spezifischen Sinnerfahrungen Subjekte mit Gewalt machen; und das heißt: ob sie diese aktiv ausüben oder ihr passiv unterworfen sind. Die Möglichkeit der Gewalt wird hier im Sinne einer „Experientialie“ (64) der Offenheit und Verletzlichkeit des Subjekts gedeutet. Doch ist es diese Antwort, die wir erwarten, wenn wir danach fragen, wie Gewalt gegen den Anderen möglich ist?

Eine spezifischere Antwort auf seine Frage entwickelt Staudigl dort, wo er dem Möglichkeitsbegriff eine ethische Wende gibt und aufzeigt, wie das Selbstverhältnis der Subjekte, also die Art und Weise, wie sie ihre Nicht-Identität und Verletzlichkeit anerkennen, direkt mit der Ausübung von Gewalt verbunden ist. Hier wird der Sinn der Gewalt mit spezifischen Begehrensformen und Affekten verbunden, mit denen Subjekte auf ihre leibhaften Desintegrationserfahrungen reagieren. So ist es nach Staudigl letztlich das Begehren nach Erlösung von der eigenen Nicht-Identität, das Subjekte für die Gewalt anfällig mache. Exemplarisch verdeutlicht er diese These am Beispiel des Rassismus und der exzessiven kollektiven Gewalt, in denen er auf der Ebene der ,Täter_innen‘ eine Form der „Alteration des Eigenen“ (228) am Werk sieht. Gemeint ist damit die Angst, nicht mit sich selbst identisch zu sein, die Subjekte umtreibt und sie dazu verleitet, diese Angst vor dem Nicht-Eigenen auf andere zu projizieren. Diese Figur bildet nach Staudigl die Basis für alle politischen Mythen und Phantasmen einer reinen und vollständigen „Verkörperung des Wir“ (227), in denen die Angst der Subjekte „ausgebeutet“ (237) wird. Solche Phantasmen führen zu Abwertungen und Ausschlüssen der Anderen, die sich bis zum Genozid steigern können. Für Staudigl ist darum die Anerkennung der Verletzlichkeit und Nicht-Identität jedes Subjekts entscheidend, um allen Formen der Gewalt den Boden zu entziehen. Die Subjekte vor der Gewalt zu schützen bedeute, so Staudigl, eine „Politik des verletzlichen Körpers“(285) zu verfolgen.

An dieser Stelle verstärkt sich allerdings der Zweifel, ob die Frage nach den Möglichkeitsbedingungen der Gewalt tatsächlich nur im Rekurs auf die leibhaften Erfahrungen von Subjekten beantwortet werden kann. Zwar ist Staudigls Vorschlag überzeugend, dass Gewalt als Sinnerfahrung mit den subjektiven Existenzbedingungen der Leibhaftigkeit und Prekarität der Identität zusammengedacht werden müsse. Die Tatsache aber, dass aus einer solchen (Angst-)Erfahrung politisch Kapital zu schlagen ist, verweist auf ein komplexeres Verständnis von Gewalt und den damit verbundenen Möglichkeitsbedingungen, die nicht nur phänomenologisch und ethisch, sondern auch machttheoretisch zu verstehen wären. Nicht zufällig bezeichnet Staudigl selbst die aktiv ausgeübte Gewalt 
als „Verletzungsmacht“ (189). Wie also ist diese Verletzungsmacht möglich? Um solche Machtphänomene zu beschreiben, müsste der enge Rahmen der phänomenologischen Gewaltbestimmung gesprengt werden, und es müssten Dimensionen sozialer Praxis einbezogen werden, die spezifisch auf den Machtaspekt der Gewalt fokussieren. Dazu gehört natürlich zentral, dass das relationale Gefüge zwischen Opfern, Täter_innen und Zeug_innen der Gewalt genauer danach analysiert wird, was sie miteinander verbindet und voneinander unterscheidet; wie sie relational aufeinander bezogen sind; ob sie tatsächlich ein einheitliches Phänomen der Gewalt konstituieren - oder nicht vielmehr deren Dissoziation und soziale Widersprüchlichkeit exemplifizieren. So wäre auch zu fragen, ob der Sinn der Gewalt nicht dekonstruktiver gefasst werden müsste, als dies die Darstellung nahelegt, weil Gewalt doch auch - und darauf weist Staudigl selbst, wenn auch eher en passant, hin - als sinnhaftes Phänomen den „Prozess lebendiger Sinnbildung [...] zerstört“ (48-49). Damit wird Gewalt als ein sozial sinnhafter und sozial destruktiver Sinnausdruck umschrieben, ohne dass dieser zentralen Aporie vertieft nachgegangen wird. Entsprechend bleibt nach der Lektüre des Buches der Eindruck, dass Gewalt zwar als multiperspektives Phänomen dargestellt wird, worin die Erfahrungen von Täter_innen, Opfern und Zeug_innen der Gewalt kategorial unterschieden werden, dass aber unklar ist, woher sich die Unterscheidung dieser drei Kategorien selbst herleitet, ob ihre Perspektiven für das Verständnis von Gewalt gleichwertig sind und wie sich Gewalt als Verhältnis zwischen Tuenden und Erleidenden beschreiben lässt.

Da sich Staudigl am Anfang und am Ende seines Buches vehement gegen Gewalttheorien abgrenzt, in denen Gewalt in ihrer instrumentellen, ,sinnlosen und sozial destruktiven Dimension thematisiert wird (Kap. 1 und 6.1), schneidet er sich den Weg ab, um den oben genannten Fragen, die unter anderem auch mit machttheoretischen Effekten und handlungstheoretischen Zwecken der Gewalt verbunden sind, weiter nachzugehen. Der eher unproduktive Gegensatz zwischen einer phänomenologischen und einer ,ursächlichen' Gewalttheorie verunmöglicht auch, dass sich die Rekonstruktion des Sinns von Gewalt mit Machtanalysen verbindet, in denen die Möglichkeit der Gewalt stärker mit sozialen und politischen Ermöglichungsbedingungen verbunden wird.

Diese Desiderate stellen die Thesen von Staudigl nicht per se in Frage, und tatsächlich sind seine Rekonstruktionen zum Zusammenhang von Gewalt und leibhafter Existenz höchst plausibel, wenn auch durch die ausufernde Fülle der verwendeten Zitate und Referenzen manche wichtigen Gedanken zu oberflächlich dargestellt werden. Die monierte Lücke fällt jedoch stärker ins Gewicht, wenn man Staudigls Anspruch ernst nimmt, wonach es ihm darum geht, eine ,integrative Theorie“ (22) zu entwickeln, die dem Zusammenhang zwischen verschiedenen Gewaltformen nachgeht und in einer „relationalen Phänomenologie“ (255) 
der Gewalt mündet. Diese sehr grundsätzlichen Ansprüche werden im Buch letztlich nicht eingelöst, da entscheidende Aspekte der Relationalität der Gewalt in ihrer machttheoretischen Dimension und sinnhaften Widersprüchlichkeit ungeklärt bleiben. Dies gilt nicht zuletzt auch für Staudigls saloppe und äußerst knapp gehaltene Abgrenzung von jenen Gewalttheorien, die sich für die Überwindung sozialer und politischer Gewaltverhältnisse einsetzen. Staudigl polemisiert hier gegen jene „Selbstgerechtigkeit einer ihre eigene Gewalt leugnenden Vernunft“, die vorgibt, Gewalt aufheben zu können (172), und fordert stattdessen, die Unüberwindbarkeit der Gewalt im Sozialen anzuerkennen. Dass er mit dieser Kritik auf Arendt Bezug nimmt, wirkt mehr als unfair - geht es Arendt doch wie Staudigl selbst auch darum, Politik von den Fantasien souveräner Herrschaft zu lösen und das Subjekt in seiner unhintergehbaren Abhängigkeit von anderen ins Zentrum zu stellen. Damit Staudigls Thesen von der Irreduzibilität der Gewalt im Sozialen mehr wären als ein Schlagwort, müsste er genauer aufzeigen, worin diese Irreduzibilität begründet ist, welche Formen der Gewalt sie tatsächlich betrifft, und warum eine Politik, die Gewalt überwinden will, zur Gewalt beiträgt. 